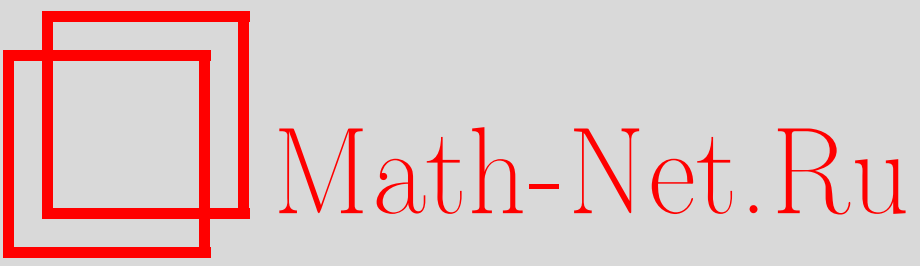

О. К. Пашаев, М. Л. Франсиско, Вырожденный четырехсолитонный виртуальный резонанс для уравнения КП-II, ТМФ, 2005, том 144, номер 1, 162-170

DOI: https://doi.org/10.4213/tmf1842

Использование Общероссийского математического портала Math-Net.Ru подразумевает, что вы прочитали и согласны с пользовательским соглашением

http://www . mathnet.ru/rus/agreement

Параметры загрузки:

IP : 3.91 .87 .62

26 апреля 2023 г., 13:56:42 
ТЕОРЕТИЧЕСКАЯ

И МАТЕМАТИЧЕСКАЯ

ФИЗИКА

Том 144, № 1

июль, 2005

(C) 2005 г.

О.К. Пашаев* , М. Л. Франсиско*

\section{ВЫРОЖДЕННЫЙ ЧЕТЫРЕХСОЛИТОННЫЙ ВИРТУАЛЬНЫЙ РЕЗОНАНС ДЛЯ УРАВНЕНИЯ КП-ІІ}

Предложен метод решения $(2+1)$-мерного уравнения Кадомцева-Петвиашвили с отрицательной дисперсией (КП-II), основанный на использовании второго и третьего членов диссипативного варианта иерархии Абловица-Каупа-Ньюэлла-Сегура (AKHC). Показано, что диссипативные солитоны (диссипатоны) соответствующих уравнений приводят к плоским солитонам уравнения КП-ІІ. На основе билинейного представления Хироты для потоков $S L(2, \mathbb{R})$-иерархии АKНС выписано новое билинейное представление для уравнения КП-II, с помощью которого построены одно- и двухсолитонные решения и исследован резонансный характер их взаимодействия. С помощью указанного билинейного представления удалось впервые построить резонансное решение с четырьмя виртуальными солитонами для уравнения КП-II и показать, что такое решение может быть получено как редукция четырехсолитонного решения в билинейном виде Хироты-Сацумы для уравнения КП-ІІ.

Ключевые слова: диссипативный солитон, иерархия Абловица-Каупа-Ньюэлла-Сегура, уравнение Кадомцева-Петвиашвили, метод Хироты, солитонный резонанс, система реакции-диффузии.

\section{1. ВВЕДЕНИЕ}

Диссипативньй вариант иерархии Абловица-Каупа-Ньюэлла-Сегура (АКНС) [1] был рассмотрен недавно в связи с $(1+1)$-мерными гравитационными моделями (гравитационными моделями на линии) [2]. Было обнаружено, что второй поток, описываемый диссипативным вариантом нелинейного уравнения Шредингера (НУШ) (так называемая система реакшии-диффузии (РД)), допускает новый тип солитонных решений, называемых диссипатонами. Диссипатоны имеют экспоненциально возрастаюшую/убываюшую амплитуды, при этом их билинейное произведение имеет форму идеального солитона, а взаимодействие носит резонансный характер.

В настоящей работе мы исследуем резонансные диссипативные солитоны в иерархии $\mathrm{AKHC} \mathrm{и} \mathrm{показьваем,} \mathrm{что} \mathrm{они} \mathrm{приводят} \mathrm{к} \mathrm{плоским} \mathrm{солитонам}(2+1)$-мерного уравнения Кадомцева-Петвиашвили с отрицательной дисперсией (КП-ІІ). В основе нашего подхода лежит следуюший метод построения решений $(2+1)$-мерного уравнения Кадомцева-Петвиашвили (КП): мы показываем, что если рассмотреть решения, удовлетворяю-

* Department of Mathematics, Izmir Institute of Technology, Urla-Izmir, 35430 Turkey. E-mail: oktaypashaev@iyte.edu.tr 
щие одновременно системам уравнений второго и третьего потоков иерархии АКНС, то их произведение $e^{+} e^{-}$удовлетворяет уравнению КП-II (см. предложение в разделе 4 ). Используя эти результаты, мы строим новое билинейное представление для уравнения KП-II, которое имеет одно- и двухсолитонное решения. Показано, что наше двухсолитонное решение соответствует вырожденному четырехсолитонному решению уравнения КП в стандартном виде Хироты и обнаруживает резонанс с четырьмя виртуальными солитонами.

\section{2. $S L(2, \mathbb{R})$-ИЕРАРХИЯ АКНС}

Диссипативная $S L(2, \mathbb{R})$-иерархия $\mathrm{AKHC} \mathrm{эволюционных} \mathrm{уравнений}$

$$
\frac{1}{2} \sigma_{3}\left(\begin{array}{l}
e^{+} \\
e^{-}
\end{array}\right)_{t_{N}}=\Re^{N+1}\left(\begin{array}{l}
e^{+} \\
e^{-}
\end{array}\right),
$$

где $N=0,1,2, \ldots, \Lambda<0$, порождается оператором рекурсии

$$
\Re=\left(\begin{array}{cc}
\partial_{x}-\frac{\Lambda}{4} e^{+} \int^{x} e^{-} & -\frac{\Lambda}{4} e^{+} \int^{x} e^{+} \\
-\frac{\Lambda}{4} e^{-} \int^{x} e^{-} & \partial_{x}+\frac{\Lambda}{4} e^{-} \int^{x} e^{+}
\end{array}\right) .
$$

Второй и третий члены иерархии АКНС выглядят соответственно как

$$
\left\{\begin{aligned}
e_{t_{1}}^{+} & =e_{x x}^{+}+\frac{\Lambda}{4} e^{+} e^{-} e^{+} \\
-e_{t_{1}}^{-} & =e_{x x}^{-}+\frac{\Lambda}{4} e^{+} e^{-} e^{-}
\end{aligned}\right.
$$

$$
\left\{\begin{array}{l}
e_{t_{2}}^{+}=e_{x x x}^{+}+\frac{3 \Lambda}{4} e^{+} e^{-} e_{x}^{+} \\
e_{t_{2}}^{-}=e_{x x x}^{-}+\frac{3 \Lambda}{4} e^{+} e^{-} e_{x}^{-}
\end{array}\right.
$$

Первая система (3), диссипативный вариант НУШ, называется системой Р Д [2]. Она связана с калибровочной формулировкой $(1+1)$-мерной гравитации, с поверхностями постоянной кривизны в псевдоевклидовом пространстве [2] и с задачей о солитоне НУШ в квантовом потенщиале [2], [3].

\section{3. РЕЗОНАНСНЫЕ ДИССИПАТОНЫ В ИЕРАРХИИ АКНС}

3.1. Диссипатоны системы уравнений $\mathbf{P}$ Д. Второй член иерархии AKHC (3), уравнение Р Д, с помощью подстановки

$$
e^{ \pm}=\sqrt{\frac{8}{-\Lambda}} \frac{G^{ \pm}(x, t)}{F(x, t)}
$$

можно записать в билинейном представлении Хироты, $t \equiv t_{1}$ :

$$
\left( \pm D_{t}-D_{x}^{2}\right)\left(G^{ \pm} \cdot F\right)=0, \quad D_{x}^{2}(F \cdot F)=-2 G^{+} G^{-}
$$


Таким образом, любое решение последней системы определяет решение системы Р Д (3). Простейшее решение билинейной системы (6) имеет вид [3]

$$
G^{ \pm}= \pm e^{\eta_{1}^{ \pm}}, \quad F=1+\frac{e^{\left(\eta_{1}^{+}+\eta_{1}^{-}\right)}}{\left(k_{1}^{+}+k_{1}^{-}\right)^{2}},
$$

где $\eta_{1}^{ \pm}=k_{1}^{ \pm} x \pm\left(k_{1}^{ \pm}\right)^{2} t+\eta_{1}^{ \pm(0)}$. Это решение определяет солитоноподобные решения системы Р Д с экспоненциально возрастающей и убывающей амплитудами, называемые диссипатонами [2]. Однако произведение $e^{+} e^{-}$имеет идеальную односолитонную форMy:

$$
e^{+} e^{-}=\frac{8 k^{2}}{\Lambda \operatorname{ch}^{2}\left[k\left(x-v t-x_{0}\right)\right]},
$$

амплитуду $k=\left(k_{1}^{+}+k_{1}^{-}\right) / 2$ и распространяется со скоростью $v=-\left(k_{1}^{+}-k_{1}^{-}\right)$из начального положения $x_{0}=-\ln \left(k_{1}^{+}+k_{1}^{-}\right)^{2}+\eta_{1}^{+(0)}+\eta_{1}^{-(0)}$.

Система РД допускает геометрическую интерпретацию на языке поверхностей постоянной кривизны [2]. Если функции $e^{ \pm}$удовлетворяют уравнениям РД (3), то соответствуюшая риманова метрика описывает двумерное псевдориманово пространство-время постоянной кривизны $\Lambda: R=g^{\mu \nu} R_{\mu \nu}=\Lambda$. Метрика, вычисленная для решения с одним диссипатоном (7), имеет сингулярность (изменение знака) при th $k(x-$ $v t)= \pm v / 2 k$. Эта сингулярность (называемая причинной) допускает физическое истолкование в рамках физики черных дыр и связана с резонансными свойствами солитонов. Действительно, построив решение с двумя диссипатонами, мы обнаруживаем, что оно описывает столкновение последних, в результате чего образуется резонансное (метастабильное) связанное состояние [3].

3.2. Диссипативные солитоны для уравнений третьего потока. Для третьего потока иерархии АКНС мы имеем систему с кубической дисперсией (4). Билинейное представление этой системы уравнений для функций $e^{ \pm}(x, t)$ в терминах трех вещественных функций $G^{ \pm}, F(5)$ имеет вид

$$
\left(D_{t}+D_{x}^{3}\right)\left(G^{ \pm} \cdot F\right)=0, \quad D_{x}^{2}(F \cdot F)=-2 G^{+} G^{-} .
$$

Из последнего уравнения получаем выражение для произведения

$$
U=e^{+} e^{-}=\frac{8}{-\Lambda} \frac{G^{+} G^{-}}{F^{2}}=\frac{4}{\Lambda} \frac{D_{x}^{2}(F \cdot F)}{F^{2}}=\frac{8}{\Lambda} \frac{\partial^{2}}{\partial x^{2}} \ln F
$$

Простейшее решение этой системы

$$
G^{ \pm}= \pm e^{\eta_{1}^{ \pm}}, \quad F=1+\frac{e^{\left(\eta_{1}^{+}+\eta_{1}^{-}\right)}}{\left(k_{1}^{+}+k_{1}^{-}\right)^{2}},
$$

где $\eta_{1}^{ \pm}=k_{1}^{ \pm} x-\left(k_{1}^{ \pm}\right)^{3} t+\eta_{1}^{ \pm(0)}$, определяет диссипативное односолитонное решение системы (4):

$$
e^{ \pm}= \pm \sqrt{\frac{8}{-\Lambda}} \frac{\left|k_{11}^{+-}\right|}{2} \frac{e^{ \pm\left(\eta_{1}^{+}-\eta_{1}^{-}\right) / 2}}{\operatorname{ch}\left[\left(k_{1}^{+}+k_{1}^{-}\right)\left(x-v t-x_{0}\right) / 2\right]},
$$




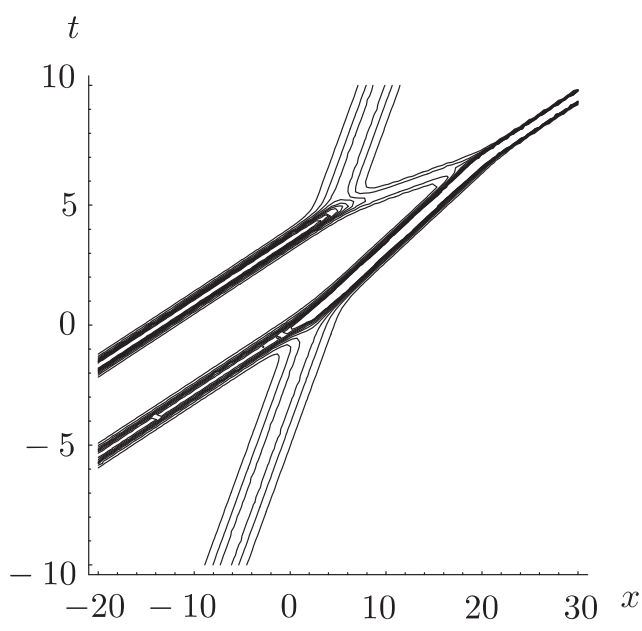

Рис. 1

где $v=k_{1}^{+2}-k_{1}^{+} k_{1}^{-}+k_{1}^{-2}, x_{0}=\left(\eta_{1}^{+(0)}+\eta_{1}^{-(0)}\right) / k_{1}^{+} k_{1}^{-}, \phi_{11}=-2 \ln k_{11}^{+-}$. Резонансное взаимодействие слияния трех диссипатонов в два диссипатона для системы (4) представлено на рис. 1.

Система (4) допускает следующую редукцию симметрии: $e^{+}=e^{-}=u$, приводящую к модифицированному уравнению Кортевега-де Фриза (мКдФ):

$$
u_{t_{2}}=u_{x x x}+\frac{3 \Lambda}{4} u^{2} u_{x}
$$

При такой редукции $k_{1}^{+}=k_{1}^{-} \equiv k$, и диссипативный солитон (12) преврашается в односолитонное решение мКд $\Phi$ :

$$
e^{+}=e^{-}=u(x, t)=\sqrt{\frac{8}{-\Lambda}} \frac{|k|}{\operatorname{ch} k\left(x-k^{2} t-x_{0}\right)} .
$$

Таким способом можно убедиться, что диссипативный солитон является достаточно общим объектом, который допускает редукцию к настоящему солитону мКдФ. Аналогичным образом диссипативное двухсолитонное решение системы (4) с помощью редукции $k_{1}^{+}=k_{1}^{-}, k_{2}^{+}=k_{2}^{-}$сводится к двухсолитонному решению мКд $\Phi$. Естественный вопрос заключается в том, чтобы найти эволюционное уравнение для произведения диссипатонов $e^{+} e^{-}$. Как будет показано ниже, таким уравнением является уравнение КП-II в размерности $(2+1)$.

\section{4. РЕЗОНАНСНЫЕ СОЛИТОНЫ УРАВНЕНИЯ КП-II}

4.1. Уравнение KП-II и иерархия АКНС. Иерархия АКНС позволяет также развить новый метод для нахождения решений $(2+1)$-мерного уравнения КП. В зависимости от знака дисперсии различают два типа уравнений КП. Знак минус в правой части уравнения КП отвечает случаю отрицательной дисперсии, а соответствующее уравнение называется КП-II. Чтобы связать уравнения КП-II с иерархией АKHC, рассмотрим пару функций $e^{+}(x, y, t), e^{-}(x, y, t)$, которые удовлетворяют уравнениям, составляюшим второй и третий члены диссипативной иерархии АКНС. Обозначим по-новому 
временны́е переменные в этих уравнениях: $t_{1} \equiv y$ и $t_{2} \equiv t$. Дифференцируя уравнения систем (3) и (4) соответственно по $t$ и по $y$, находим, что эти системы совместимы.

ПрЕДЛОЖЕНИЕ. Пусть функции $e^{+}(x, y, t) u e^{-}(x, y, t)$ являются одновременно решениями уравнений (3) и (4). Тогда функиия $U(x, y, t) \equiv e^{+} e^{-}$удовлетворяет уравнению КП-II

$$
\left(4 U_{t}+\frac{3 \Lambda}{4}\left(U^{2}\right)_{x}+U_{x x x}\right)_{x}=-3 U_{y y}
$$

ДОКАЗАТЕЛЬСТВО. Возьмем производную от функции $U$ по переменной $y$ и используем формулы $(3)$, так что $U_{y}=\left(e_{x}^{+} e^{-}-e_{x}^{-} e^{+}\right)_{x}$,

$$
U_{y y}=\left(e_{x x x}^{+} e^{-}+e_{x x x}^{-} e^{+}-\left(e_{x}^{+} e_{x}^{-}\right)_{x}\right)+\frac{\Lambda}{2} U_{x} U
$$

Аналогичным образом для $U_{t}$ получаем

$$
\begin{aligned}
U_{t} & =-\left(e_{x x x}^{+} e^{-}+\frac{3 \Lambda}{4} U e^{-} e_{x}^{-}+e_{x x x}^{-} e^{+}+\frac{3 \Lambda}{4} U e_{x}^{-} e^{+}\right), \\
U_{x t} & =-\left(e_{x x x}^{+} e^{-}+e_{x x x}^{-} e^{+}+\frac{3 \Lambda}{4} U U_{x}\right)_{x} .
\end{aligned}
$$

Объединяя полученные формулы,

$$
4 U_{x t}+3 U_{y y}=\left[-e_{x x x}^{+} e^{-}-e_{x x x}^{-} e^{+}-\frac{3 \Lambda}{2} U U_{x}-3\left(e_{x}^{+} e_{x}^{-}\right)_{x}\right]_{x},
$$

и используя формулу $U_{x x x}=e_{x x x}^{+} e^{-}+e_{x x x}^{-} e^{+}+3 e_{x x}^{+} e_{x}^{-}+3 e_{x}^{+} e_{x x}^{-}$, получаем уравнение KП-II $(15)^{1)}$.

4.2. Билинейное представление для уравнения КП-ІІ с помощью потоков АКНС. Используя билинейные представления для систем (3) и (4) и доказанное выше предложение, можно найти билинейное представление для уравнения КП-II. Для системы Р Д (3) билинейное представление дается формулами (6), а для системы (4) третьего потока - формулами (9).

Рассмотрим теперь $G^{ \pm}$и $F$ как функции трех переменных $G^{ \pm}=G^{ \pm}(x, y, t), \quad F=$ $F(x, y, t)$ и потребуем, чтобы они были одновременно решениями билинейных систем (6) и (9). Поскольку второе уравнение в обеих системах одинаковое, достаточно рассмотреть следуюшую билинейную систему:

$$
\left\{\begin{array}{l}
\left( \pm D_{y}-D_{x}^{2}\right)\left(G^{ \pm} \cdot F\right)=0 \\
\left(D_{t}+D_{x}^{3}\right)\left(G^{ \pm} \cdot F\right)=0 \\
D_{x}^{2}(F \cdot F)=-2 G^{+} G^{-}
\end{array}\right.
$$

\footnotetext{
1) Как недавно указал нам Б. Конопельченко, подобные результаты известны также в литературе как симметрийные редукции КП (см., например, [4]).
} 
Тогда в соответствии с предложением любое решение этой системы порождает решение уравнения КП-ІІ. Из последнего уравнения можно прямо выразить функцию $U$ только через функцию $F$ :

$$
U=e^{+} e^{-}=\frac{8}{-\Lambda} \frac{G^{+} G^{-}}{F^{2}}=\frac{4}{\Lambda} \frac{D_{x}^{2}(F \cdot F)}{F^{2}}=\frac{8}{\Lambda} \frac{\partial^{2}}{\partial x^{2}} \ln F .
$$

Простейшее решение этой системы

$$
G^{ \pm}= \pm e^{\eta_{1}^{ \pm}}, \quad F=1+\frac{e^{\left(\eta_{1}^{+}+\eta_{1}^{-}\right)}}{\left(k_{1}^{+}+k_{1}^{-}\right)^{2}}
$$

где $\eta_{1}^{ \pm}=k_{1}^{ \pm} x \pm\left(k_{1}^{ \pm}\right)^{2} y-\left(k_{1}^{ \pm}\right)^{3} t+\eta_{1}^{ \pm(0)}$, определяет односолитонное решение уравнения КП-ІІ по формуле (21):

$$
U=\frac{2\left(k_{1}^{+}+k_{1}^{-}\right)^{2}}{\Lambda \operatorname{ch}^{2}\left[\left(\left(k_{1}^{+}+k_{1}^{-}\right) x+\left(k_{1}^{+2}-k_{1}^{-2}\right) y-\left(k_{1}^{+3}+k_{1}^{-3}\right) t+\gamma\right) / 2\right]},
$$

где $\gamma=-\ln \left(k_{1}^{+}+k_{1}^{-}\right)^{2}+\eta_{1}^{+(0)}+\eta_{1}^{-(0)}$. Этот солитон представляет собой плоский волновой барьер, двигающийся в произвольном направлении, и называется плоским солитоном.

4.3. Двухсолитонное решение. Продолжая разложение Хироты, находим двухсолитонное решение в виде

$$
\begin{gathered}
G^{ \pm}= \pm\left(e^{\eta_{1}^{ \pm}}+e^{\eta_{2}^{ \pm}}+\alpha_{1}^{ \pm} e^{\eta_{1}^{+}+\eta_{1}^{-}+\eta_{2}^{ \pm}}+\alpha_{2}^{ \pm} e^{\eta_{2}^{+}+\eta_{2}^{-}+\eta_{1}^{ \pm}}\right), \\
F=1+\frac{e^{\eta_{1}^{+}+\eta_{1}^{-}}}{\left(k_{11}^{+-}\right)^{2}}+\frac{e^{\eta_{1}^{+}+\eta_{2}^{-}}}{\left(k_{12}^{+-}\right)^{2}}+\frac{e^{\eta_{2}^{+}+\eta_{1}^{-}}}{\left(k_{21}^{+-}\right)^{2}}+\frac{e^{\eta_{2}^{+}+\eta_{2}^{-}}}{\left(k_{22}^{+-}\right)^{2}}+\beta e^{\eta_{1}^{+}+\eta_{1}^{-}+\eta_{2}^{+}+\eta_{2}^{-}}, \\
\text {где } \eta_{i}^{ \pm}=k_{i}^{ \pm} x \pm\left(k_{i}^{ \pm}\right)^{2} y-\left(k_{i}^{ \pm}\right)^{3} t+\eta_{i}^{ \pm(0)}, k_{i j}^{a b}=k_{i}^{a}+k_{j}^{b}, i, j=1,2, a, b=+,-, \\
\alpha_{1}^{ \pm}=\frac{\left(k_{1}^{ \pm}-k_{2}^{ \pm}\right)^{2}}{\left(k_{11}^{+-} k_{21}^{ \pm \mp}\right)^{2}}, \quad \alpha_{2}^{ \pm}=\frac{\left(k_{1}^{ \pm}-k_{2}^{ \pm}\right)^{2}}{\left(k_{22}^{+-} k_{12}^{ \pm \mp}\right)^{2}}, \quad \beta=\frac{\left(k_{1}^{+}-k_{2}^{+}\right)^{2}\left(k_{1}^{-}-k_{2}^{-}\right)^{2}}{\left(k_{11}^{+-} k_{12}^{+-} k_{21}^{+-} k_{22}^{+-}\right)^{2}} .
\end{gathered}
$$

Это дает всюду регулярное двухсолитонное решение уравнения КП-ІІ по формуле (21).

4.4. Вырожденное четырехсолитонное решение. Для уравнения КП-ІІ известно другое билинейное представление исключительно в терминах функции $F[5]$ :

$$
\left(D_{x} D_{t}+D_{x}^{4}+D_{y}^{2}\right)(F \cdot F)=0
$$

поэтому естественно сравнить солитонные решения наших билинейных уравнений (20) с аналогичными решениями последнего уравнения. Чтобы решить уравнение (26), рассмотрим $F=1+\varepsilon F_{1}+\varepsilon^{2} F_{2}+\cdots$. Решение $F_{1}=e^{\eta_{1}}$, где $\eta_{1}=k_{1} x+\Omega_{1} y+\omega_{1} t+\eta_{1}^{0}$, дисперсионное соотношение имеет вид $k_{1} \omega_{1}+k_{1}^{4}+\Omega_{1}^{2}=0$ и $F_{n}=0, n=2,3, \ldots$, при 
отож дествлениях $k_{1}=k_{1}^{+}+k_{1}^{-}, \Omega_{1}=\sqrt{3}\left(k_{1}^{+2}-k_{1}^{-2}\right), \omega_{1}=-4\left(k_{1}^{+3}+k_{1}^{-3}\right)$ и изменении масштаба $4 t \rightarrow t, \sqrt{3} y \rightarrow y$, определяет односолитонное решение КП-ІІ (15). Понятно, что оно совпадает с нашим односолитонным решением (23). Однако двухсолитонное решение уравнения (26) [6] не соответствует нашему двухсолтитонному решению (24), (25). Наличие четырех различных членов $e^{\eta_{i}^{ \pm}+\eta_{k}^{ \pm}}$в выражении $(25)$ наводит на мысль, что наше двухсолитонное решение должно соответствовать некоторому вырож денному случаю четырехсолитонного решения уравнения $(26)^{2}$. Чтобы построить четырехсолитонное решение, найдем сначала следуюшие решения билинейного уравнения (26):

$$
F_{1}=e^{\eta_{1}}, \quad F_{2}=e^{\eta_{2}}, \quad F_{4}=e^{\eta_{3}},
$$

где $\eta_{i}=k_{i} x+\Omega_{i} y+\omega_{i} t+\eta_{i}^{0}, i=1,2,3$, а дисперсионные соотношения имеют вид

$$
k_{i} \omega_{i}+k_{i}^{4}+\Omega_{i}^{2}=0
$$

и

$$
F_{3}=\alpha_{12} e^{\eta_{1}+\eta_{2}}, \quad F_{5}=\alpha_{13} e^{\eta_{1}+\eta_{3}}, \quad F_{6}=\alpha_{23} e^{\eta_{1}+\eta_{3}},
$$

где

$$
\alpha_{i j}=-\frac{\left(k_{i}-k_{j}\right)\left(\omega_{i}-\omega_{j}\right)+\left(k_{i}-k_{j}\right)^{4}+\left(\Omega_{i}-\Omega_{j}\right)^{2}}{\left(k_{i}+k_{j}\right)\left(\omega_{i}+\omega_{j}\right)+\left(k_{i}+k_{j}\right)^{4}+\left(\Omega_{i}+\Omega_{j}\right)^{2}}, \quad i, j=1,2,3 .
$$

Используем теперь для нашего решения параметризацию вида

$$
\begin{array}{lll}
k_{1}=k_{1}^{+}+k_{1}^{-}, & \omega_{1}=-4\left(k_{1}^{+3}+k_{1}^{-3}\right), & \Omega_{1}=\sqrt{3}\left(k_{1}^{+2}-k_{1}^{-2}\right), \\
k_{2}=k_{2}^{+}+k_{2}^{-}, & \omega_{2}=-4\left(k_{2}^{+3}+k_{2}^{-3}\right), & \Omega_{2}=\sqrt{3}\left(k_{2}^{+2}-k_{2}^{-2}\right), \\
k_{3}=k_{1}^{+}+k_{2}^{-}, & \omega_{3}=-4\left(k_{1}^{+3}+k_{2}^{-3}\right), & \Omega_{3}=\sqrt{3}\left(k_{1}^{+2}-k_{2}^{-2}\right), \\
k_{4}=k_{2}^{+}+k_{1}^{-}, & \omega_{4}=-4\left(k_{2}^{+3}+k_{1}^{-3}\right), & \Omega_{4}=\sqrt{3}\left(k_{2}^{+2}+k_{1}^{-2}\right),
\end{array}
$$

удовлетворяюшую дисперсионным соотношениям (28). Подставляя эти формулы параметризации в приведенные выше решения, находим

$$
\alpha_{13}=0 \Rightarrow F_{5}=0, \quad \alpha_{23}=0 \Rightarrow F_{6}=0 .
$$

Продолжая разложение Хироты с решением $F_{7}=e^{\eta_{4}}$, где $\eta_{4}=k_{4} x+\Omega_{4} y+\omega_{4} t+\eta_{4}^{0}$, находим, что $F_{8}=\alpha_{14} e^{\eta_{1}+\eta_{4}}$, где

$$
\alpha_{14}=-\frac{\left(k_{1}-k_{4}\right)\left(\omega_{1}-\omega_{4}\right)+\left(k_{1}-k_{4}\right)^{4}+\left(\Omega_{1}-\Omega_{4}\right)^{2}}{\left(k_{1}+k_{4}\right)\left(\omega_{1}+\omega_{4}\right)+\left(k_{1}+k_{4}\right)^{4}+\left(\Omega_{1}+\Omega_{4}\right)^{2}} .
$$

С учетом выбранной параметризации (31) последнее решение также обрашается в нуль:

$$
\alpha_{14}=0 \Rightarrow F_{8}=0
$$

\footnotetext{
${ }^{2)}$ Авторы благодарны Дж. Хиетаринта, высказавшему это предположение.
} 
Следуюшее решение $F_{9}=\alpha_{24} e^{\eta_{2}+\eta_{4}}$, где

$$
\alpha_{24}=-\frac{\left(k_{2}-k_{4}\right)\left(\omega_{2}-\omega_{4}\right)+\left(k_{2}-k_{4}\right)^{4}+\left(\Omega_{2}-\Omega_{4}\right)^{2}}{\left(k_{2}+k_{4}\right)\left(\omega_{2}+\omega_{4}\right)+\left(k_{2}+k_{4}\right)^{4}+\left(\Omega_{2}+\Omega_{4}\right)^{2}},
$$

также равно нулю:

$$
\alpha_{24}=0 \quad \Rightarrow \quad F_{9}=0 .
$$

Далее имеем $F_{10}=0$ и $F_{11}=\alpha_{34} e^{\eta_{3}+\eta_{4}}$, где

$$
\alpha_{34}=-\frac{\left(k_{3}-k_{4}\right)\left(\omega_{3}-\omega_{4}\right)+\left(k_{3}-k_{4}\right)^{4}+\left(\Omega_{3}-\Omega_{4}\right)^{2}}{\left(k_{3}+k_{4}\right)\left(\omega_{3}+\omega_{4}\right)+\left(k_{3}+k_{4}\right)^{4}+\left(\Omega_{3}+\Omega_{4}\right)^{2}} .
$$

Дальнейшая проверка для членов более высокого порядка дает $F_{12}=F_{13}=\cdots=0$. Таким образом, мы имеем вырожденное четырехсолитонное решение уравнения (26) вида

$$
F=1+e^{\eta_{1}}+e^{\eta_{2}}+e^{\eta_{3}}+e^{\eta_{4}}+\alpha_{12} e^{\eta_{1}+\eta_{2}}+\alpha_{34} e^{\eta_{3}+\eta_{4}} .
$$

Сравнивая это решение с решением (25) и учитывая, что в соответствии с используемой параметризацией (31) $\eta_{1}+\eta_{2}=\eta_{3}+\eta_{4}$, видим, что решения совпадают. Проведенное рассмотрение показывает, что наше двухсолитонное решение уравнения КП-II может быть получено редукцией четырехсолитонного решения уравнения в каноническом виде Хироты (26). Более того, оно позволяет найти новый резонанс с четырьмя виртуальными солитонами для уравнения КП-ІІ.

4.5. Резонансное взаимодействие плоских солитонов. Выбирая различные значения параметров для нашего двухсолитонного решения, мы обнаруживаем, что взаимодействие солитонов имеет резонансный характер. При значениях параметров $k_{1}^{+}=$ $2, k_{1}^{-}=1, k_{2}^{+}=1, k_{2}^{-}=0.3$ в случае равенства нулю констант, определяющих пространственные сдвиги, мы получаем движушееся в плоскости двухсолитонное решение, образуюшее четыре так называемых виртуальных солитона (солитоны без асимптотических состояний на бесконечности) (рис. 2).

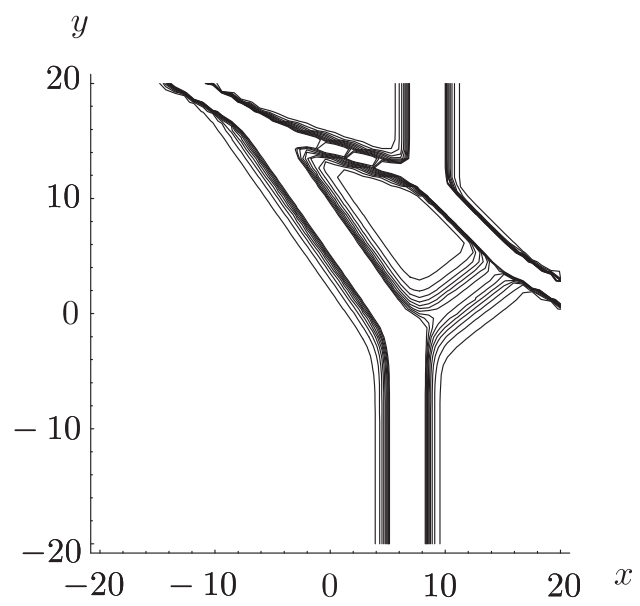

Рис. 2 
Резонансный характер взаимодействия наших плоских солитонов связан с резонансной природой рассмотренных в разделе 3 диссипатонов. Это было также отмечено в ряде систем, однако, насколько известно авторам, резонанс с образованием четырех виртуальных солитонов для уравнения КП-II [7] получен в настоящей работе впервые. На семинаре "Нелинейная физика. Теория и эксперимент. III" авторы узнали, что совсем недавно Биондини и Кодама, используя теорию Сато, также построили резонансные солитоны для уравнения КП-II [8]. Сравнение показало, что наше билинейное ограничение играет ту же роль, что и цепочка Тоды в работе этих авторов.

\section{5. ЗАКЛЮЧЕНИЕ}

Идея использования пары уравнений иерархии АКНС для построения решений уравнения КП может быть применена также к многомерным системам со структурой нулевой кривизны, таким как калибровочная теория Черна-Саймонса. Наше трехмерное представление нулевой кривизны уравнения КП-ІІ дает плоскую неабелеву связность для $S L(2, \mathbb{R})$ и соответствует сектору трехмерной гравитационной теории. Недавно мы показали, что идея, аналогичная представленной в настояшей работе, может быть применена также к иерархии Каупа-Ньюэлла. В этом случае, объединяя второй и третий потоки диссипативного варианта НУШ с производной, мы обнаружили резонансную солитонную динамику для модифицированного уравнения КП-II [9].

Благодарности. Авторы благодарны Б. Конопельченко, А. Погребкову и Д. Биондини за полезные замечания и Ю. Кодаме за многочисленные ценные обсуждения, проясняющие полученные нами результаты. Эта работа была частично поддержана Izmir Institute of Technology, Izmir, Turkey.

\section{Список литературы}

[1] M. Ablowitz, D. Kaup, A. Newell, H. Segur. Stud. Appl. Math. 1974. V. 53. P. 249.

[2] L. Martina, O.K. Pashaev, G. Soliani. Class. Q. Grav. 1997. V. 14. P. 3179; Phys. Rev. D. 1998. V. 58. P. 084025.

[3] O. K. Pashaev, J.-H. Lee. Mod. Phys. Lett. A. 2002. V. 17. № 24. P. 1601; ANZIAM J. 2002. V. 44. P. 73.

[4] B. Konopelchenko, W. Strampp. J. Math. Phys. 1992. V. 33. P. 3676; Y. Cheng, Y.-S. Li. J. Phys. A. 1992. V. 25. P. 419; C. Cao, Y. Wu, X. Geng. J. Math. Phys. 1999. V. 40. № 8. P. 3948.

[5] Р. Хирота. Прямые методы в теории солитонов. В сб.: Солитоны. Ред. Р. Буллаф, Ф. Кодри. M.: Миp, 1983. С. 175-192.

[6] K. Ohkuma, M. Wadati. J. Phys. Soc. Japan. 1983. V. 52. № 3. P. 749.

[7] E. Infeld, G. Rowlands. Nonlinear Waves, Solitons and Chaos. Cambridge: Camb. Univ. Press, 2000.

[8] G. Biondini, Y. Kodama. J. Phys. A. 2003. V. 36. P. 10519; Y. Kodama. Young diagrams and N-soliton solutions of the KP equation. nlin.SI/0406033.

[9] Дж.-Х. Ли, О.К. Пашаев. ТМФ. 2005. Т. 144. № 1. С. 133. 